\title{
Can network biology unravel the aetiology of congenital hyperinsulinism?
}

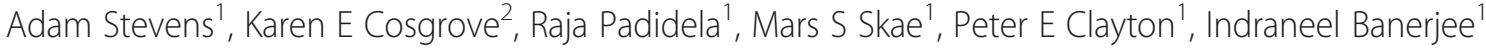 \\ and Mark J Dunne $e^{2^{*}}$
}

\begin{abstract}
Congenital Hyperinsulinism is a condition with a number of genetic causes, but for the majority of patients, the underlying aetiology is unknown. We present here a rational argument for the use of computational biology as a valuable resource for identifying new candidate genes which may cause disease and for understanding the complex mechanisms which define the pathophysiology of this rare disease.
\end{abstract}

\section{Letter}

Congenital Hyperinsulinism (CHI) is a rare disease, but is the most common cause of recurrent hypoglycaemia in infancy [1]. The treatment of CHI can be difficult and involves drugs which may not be successful and often are poorly tolerated. As a potentially life-threatening condition, CHI is associated with lifelong sequelae - including critical brain damage (epilepsy, cerebral palsy and neurological impairment) in up to $40 \%$ of cases. To date, nine candidate genes associate with $\mathrm{CHI}$, but for the majority of patients - estimated to be approximately $65 \%$, both the aetiology of the $\mathrm{CHI}$ and the mechanisms of disease are unknown.

Our current approach to the classification and treatment of CHI is based largely upon observational correlations between the pathological analysis of candidate gene defects and clinical symptoms of hypoglycaemia [1-3]. In this respect, there are similarities between $\mathrm{CHI}$ and many other diseases in which numerous mutations in different genes give rise to clinical phenotypes that are essentially indistinguishable from one another. However, under normal physiological conditions, cells function correctly because there is a high degree of interdependency between individual biochemical components (DNA, RNA, proteins and metabolites) and their complex interactions (DNA-protein interactions, protein-protein interactions, metabolic and biochemical pathways, etc.), and tissues function in a co-ordinated manner because there is

\footnotetext{
* Correspondence: mark.j.dunne@manchester.ac.uk

${ }^{2}$ Faculty of Life Sciences, University of Manchester, Manchester M13 9PT, UK Full list of author information is available at the end of the article
}

interplay between different cell types. Diseases rarely result from an abnormality in a single gene, but are in fact the manifestation of disturbances in the multiple networks that integrate cellular processes, and those that link cells with tissues, and tissues with organ systems. As a result, current approaches to molecular diagnosis, however valuable, have shortcomings. These include a lack of sensitivity in identifying preclinical disease, a poor ability to predict prognosis, and ambiguity in defining and resolving a condition where several clinical phenotypes can be observed. All of these inadequacies are evident in $\mathrm{CHI}$, with our current understanding of the causes of disease failing to distinguish transient from persistent disease at the point of presentation and to determine accurately the severity of disease. Also, it is not possible to identify at diagnosis which patients require curative surgery from those who could be successfully managed by short or long-term medical therapy. For these reasons we believe an innovative approach to $\mathrm{CHI}$ is required - one which can identify new causes and new mechanisms of dysfunction. One such approach is the use of network biology, first to summate the various interactions and interdependencies between gene networks, and second to identify critical components and pathways which may contribute to the pathophysiology of $\mathrm{CHI}$.

Today, a number of diseases are being redefined through the combination of contemporary molecular techniques (e.g. expression arrays, epi/genomic analysis, proteomic and metabolomics analysis) and bioinformatics to describe the interconnected networks that govern normal cellular function and are involved in the pathophysiology of disease [4-6]. First published in 2007, the "human disease network"
C Biomed Central

(C) 2013 Stevens et al.; licensee BioMed Central Ltd. This is an Open Access article distributed under the terms of the Creative Commons Attribution License (http://creativecommons.org/licenses/by/2.0), which permits unrestricted use, distribution, and reproduction in any medium, provided the original work is properly cited. 
describes a concept in which a specific genetic abnormality not only alters the product that it encodes, but also changes the activity of the network to which it belongs [7]. This change in activity can spread along intra- and intercellular links of a network of interconnectivity and in doing so it will also alter the activity of genes or other biochemical products that otherwise carry no abnormalities [7,8]. A consequence of this widely-held hypothesis is that the interdependencies among molecular components will form strong functional and causal relationships which can be used to map diseases in the form of "disease networks". With an estimated excess of 100,000 "nodes" representing genes, RNA, proteins, and metabolites, and an even higher number of "links" representing the functionally-relevant interactions between the nodes (including DNA-protein interactions, DNA-chemical interactions, protein-protein interactions, metabolic / biochemical pathways, etc.), the scale and complexity of this network - the "human interactome", is daunting. However, many emerging studies now describe how signature changes in the expression or activities of nodes as a consequence of disease can be integrated within the human interactome and that this relationship can then be used to unlock some of the complexities of pathophysiology [4-8]. With this in mind, we have recently analysed the bioinformatics of the nine known CHI-causing genes - which range from transcription factors to metabolic enzymes and solute transporters. This was performed by generating an interaction network seeded by the known CHI-associated genes (GLUD, SLC16A1, HADH, UCP2, KCNJ11, ABCC8, HNF1A, GCK, $H N F 4 A$ ) and their physical and genetic interactors as identified using the BioGRID interactome model [9]. Surprisingly, we found that this diverse range of genes will form a core network - described here as the CHIDisease Network, composed of highly connected members, Figure 1. There is increasing evidence that the functions associated with a biological network correlate with modular structure within the network [10]. We therefore assessed the presence of modularity in the derived $\mathrm{CHI}$ related network using spectral-partition clustering [11] and assessed known biological pathways present in these modules, Table 1.

This is the first analysis of its kind for CHI or any other monogenic disorder of glucose-regulation in infancy or childhood (e.g. neonatal diabetes mellitus, MODY, etc.). From here, we are now in a position to explore the possibility that genes integral to the network or tightly connected to it, may be new candidates for the aetiology of $\mathrm{CHI}$ and other monogenic causes of glucose-regulation disorders. Furthermore, by analysis of

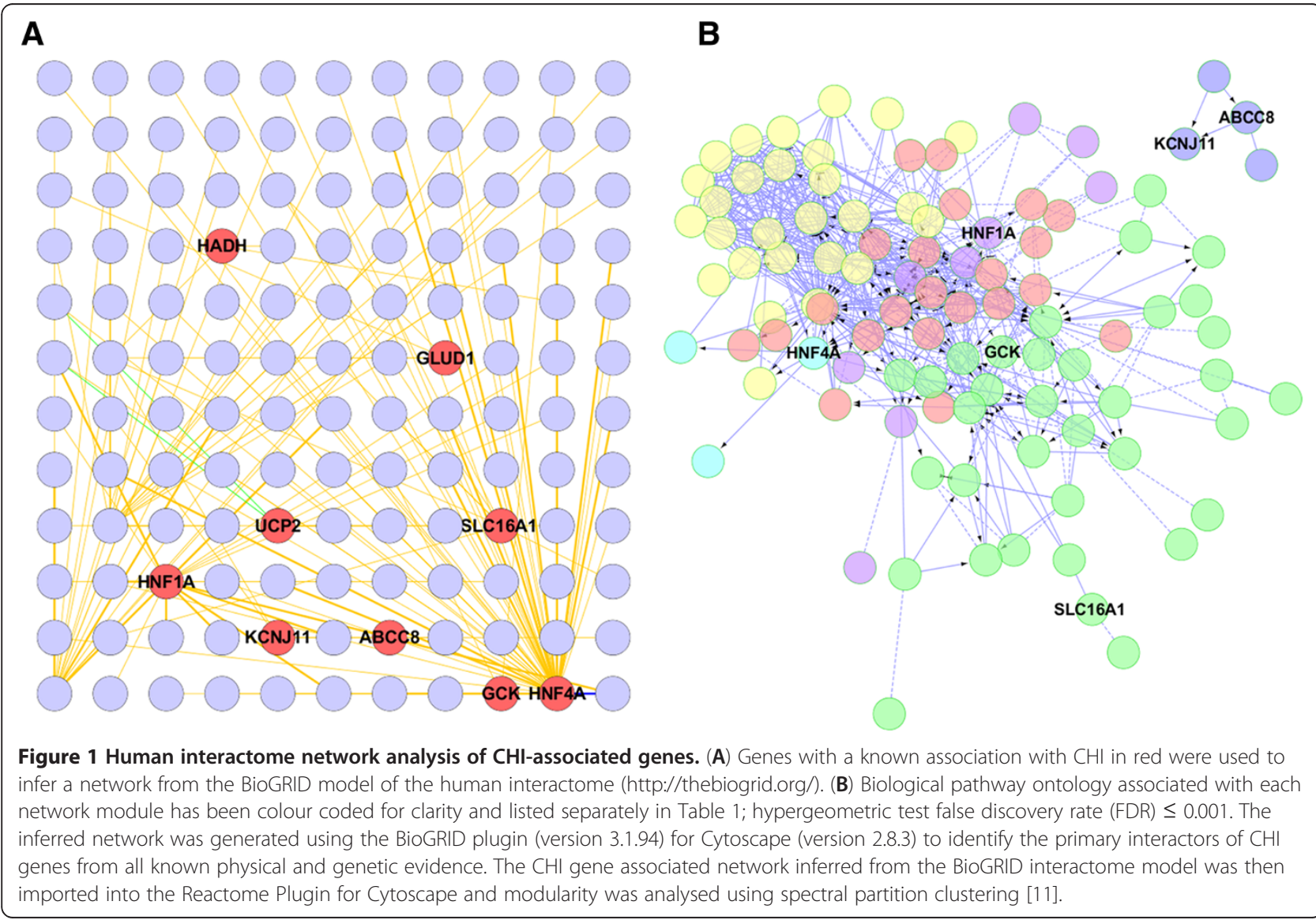


Table 1 Pathway ontology associated with the $\mathrm{CHI}$ disease network

\begin{tabular}{|c|c|c|}
\hline Network module & Biological pathway & FDR \\
\hline \multirow[t]{6}{*}{ Cellular Signalling } & Tropomyosin Receptor Kinase Signalling & $5.0 \times 10^{-5}$ \\
\hline & RAF/MAP Kinase Cascade & $5.3 \times 10^{-5}$ \\
\hline & Neurotrophin Signalling & $5.9 \times 10^{-5}$ \\
\hline & mTOR Signalling & $6.3 \times 10^{-5}$ \\
\hline & Syndecan-1-mediated Signalling & $4.0 \times 10^{-4}$ \\
\hline & TRAIL Signalling & $3.0 \times 10^{-4}$ \\
\hline \multirow[t]{4}{*}{ Nuclear Signalling } & Regulation of SMAD2/3 Signalling & $1.0 \times 10^{-4}$ \\
\hline & Oestrogen Receptor-a Signalling & $1.1 \times 10^{-4}$ \\
\hline & Oestrogen Receptor- $\beta$ Signalling & $1.5 \times 10^{-4}$ \\
\hline & Retinoic Acid Receptor Signalling & $1.4 \times 10^{-4}$ \\
\hline \multirow[t]{4}{*}{ Growth Factor Signalling } & BARD1 Signalling Events & $1.3 \times 10^{-4}$ \\
\hline & p53 Signalling pathway & $2.0 \times 10^{-4}$ \\
\hline & HDAC Class III Signalling & $1.0 \times 10^{-3}$ \\
\hline & TGF $\beta$ Signalling & $2.6 \times 10^{-4}$ \\
\hline \multirow[t]{3}{*}{ Development } & ErbB2/ErbB3 Signalling & $6.6 \times 10^{-3}$ \\
\hline & Presenilin Signalling & $9.0 \times 10^{-3}$ \\
\hline & GMCSF-Mediated Signalling & $6.6 \times 10^{-3}$ \\
\hline Function & Integration of Energy Metabolism & $1.0 \times 10^{-3}$ \\
\hline
\end{tabular}

Five network modules associate with the CHI Disease Network (Figure 1) and each module has nodes which are represented in a number of canonical biological pathways (www.ncbi.nlm.nih.gov/biosystems/). Several of these have been highlighted for each module along with their False Discovery Rate (FDR) which represents the chance occurrence of nodes being co-located with pathways.

the components of the associated pathway ontology listed in Table 1, we have created a portal to identify novel mechanisms of disease which may provide insights for the management and treatment of CHI. We strongly believe that these in silico techniques involving access to readily available databases are highly applicable to many rare diseases, and that when used alone or in combination with other datasets - e.g. metabolomic, they will form the future basis of identifying of new candidate gene defects, and understanding the pathophysiology of rare diseases.

\section{Acknowledgements}

AS initiated and undertook the bioinformatic analysis; AS, KEC, RP, MSS, IB, and PEC participated in the interpretation of data and in drafting the manuscript. MJD conceived the study, and participated in its design and coordination and helped to draft the manuscript. All authors have read and approved the final manuscript.

\section{Author details}

'Department of Endocrinology, Royal Manchester Children's Hospital, Oxford Road, Manchester M13 9WL, UK. ${ }^{2}$ Faculty of Life Sciences, University of Manchester, Manchester M13 9PT, UK.

Received: 23 November 2012 Accepted: 3 February 2013 Published: 8 February 2013

\section{References}

1. De León DD, Stanley CA: Mechanisms of disease: advances in diagnosis and treatment of hyperinsulinism in neonates. Nat Clin Pract Endocrinol Metab 2007, 3(1):57-68
2. Senniappan S, Shanti B, James C, Hussain K: Hyperinsulinaemic hypoglycaemia: genetic mechanisms, diagnosis and management. J Inherit Metab Dis 2012, 35(4):589-601.

3. Arnoux JB, Verkarre V, Saint-Martin C, Montravers F, Brassier A, Valayannopoulos V, Brunelle F, Fournet JC, Robert JJ, Aigrain Y, BellannéChantelot $C$, de Lonlay P: Congenital hyperinsulinism: current trends in diagnosis and therapy. Orphanet J Rare Dis 2011, 6:63-70.

4. Barabási AL, Gulbahce N, Loscalzo J: Network medicine: a network-based approach to human disease. Nat Rev Genet 2011, 12(1):56-68.

5. Liu YY, Slotine JJ, Barabási AL: Controllability of complex networks. Nature 2011, 473(7346):167-73.

6. Vidal M, Cusick ME, Barabási AL: Interactome networks and human disease. Cell 2011, 144(6):986-98.

7. Loscalzo J, Kohane I, Barabasi AL: Human disease classification in the postgenomic era: a complex systems approach to human pathobiology. Mol Syst Biol 2007, 3:124.

8. Goh Kl, Cusick ME, Valle D, Childs B, Vidal M, Barabási AL: The human disease network. Proc Natl Acad Sci (USA) 2006, 104(21):8685-90.

9. Chatr-Aryamontri A, Breitkreutz BJ, Heinicke S, Boucher L, Winter A, Stark C, Nixon J, Ramage L, Kolas N, O'Donnell L, Reguly T, Breitkreutz A, Sellam A, Chen D, Chang C, Rust J, Livstone M, Oughtred R, Dolinski K, Tyers M: The BioGRID interaction database: 2013 update. Nucleic Acids Res 2013, 41(D1):D816-23.

10. Zhao Y, Mooney SD: Functional organization and its implication in evolution of the human protein-protein interaction network. BMC Genomics 2012, 13(150):p1-10.

11. Newman ME: Modularity and community structure in networks. Proc Nat/ Acad Sci (USA) 2006, 103(23):8577-82 